\title{
Multitasking and performance measurement
}

\author{
by \\ Niels Nannerup \\ and \\ Kasper Krogh Olsen
}

Discussion Papers on Business and Economics

No. 20/2014

FURTHER INFORMATION Department of Business and Economics Faculty of Business and Social Sciences University of Southern Denmark Campusvej 55 DK-5230 Odense M Denmark

Tel.: +45 65503271

Fax: +456550 3237

E-mail:1ho@sam.sdu.dk http://www.sdu.dk/ivoe 


\title{
Multitasking and performance measurement
}

\author{
Niels Nannerup* Kasper Krogh Olsen*†
}

This version: December, 2014

\begin{abstract}
In a principal-agent setting, we consider a combined problem of multitasking and performance measurement. The principal can choose to reward the agent both directly for providing effort into a specific activity, and based on the outcome delivered to the principal. Both the issue of multitasking and any private knowledge the agent might possess will lead the principal to use a performance measurement more. This applies even if the measurement is poorly correlated with the actual outcome to the principal.
\end{abstract}

JEL classification: D82; D86; L51

Keywords: Multitasking; pay for performance

\section{Introduction}

Finding the right way for a principal to reward an agent is one of the most difficult problems that organizations face when designing incentive plans. As a way of helping to solve this problem the use of performance based contracts has become increasingly popular among policy-makers as the primary way of creating incentives (Burgess and Ratto, 2003; Weibel et al., 2010). This has especially been the case within the health care sector, where the Pay for Performance programs have been introduced in a number of countries (Greene and Nash, 2009; Gravelle et al., 2010). The principal thereby seeks to provide incentives to the agent to achieve a predefined collection of the 'output' (a good or service) of the production process by (at least partially) basing the agent's salary on whether or not this output has been produced. Apart from the advantage that performance based contracting rewards an agent for what he achieves and not the resources he has allocated to achieve it, it also makes the agent strive to achieve the specified performance at the smallest possible cost, and thereby make the delivery of services to the employer more efficient.

\footnotetext{
*Department of Business and Economics, University of Southern Denmark

${ }^{\dagger}$ Corresponding author. Email: kasko@sam.sdu.dk
} 
This paper focusses on performance measurements as a direct alternative to rewarding the agent a piece rate for e.g. working hours. We analyse a performance based contract in a setting with a well-known reward scheme and with three limitations to the contract; first that the principal cannot write a binding contract on some parts of the agent's work process, secondly that the agent possesses private knowledge about his own productivity, and thirdly that the principal can only base a performance contract on a distorted measurement. The principal anticipates these limitations, and incorporates them into the contract in the form of a performance measurement.

We expand the existing literature by introducing a model in which the principal has the option to reward the agent through both a direct reward for exerting effort and through a performance measurement generated by the same effort. This allows us to directly compare the two alternative payment methods in the contract and see how they each are affected by different elements of the contractual relationship. Further, we allow for private information in the agent's choice of effort level, which we show to have a significant effect on the principal's choice of wages based on effort and performance.

The choice of focus in this paper is motivated by a number of observations: The first being that even though the principal can write a binding contract on a wide range of activities that the agent can perform, she will in many cases not be able to specify every possible activity that she wants the agent to undertake in a contract, and many contracts thereby suffers from multitasking problems as described by Holmström and Milgrom (1991). The multitasking problem can both arise because the agent becomes aware of alternative input methods to the production process, compared to the method rewarded in the written contract; because the task is so indefinable that it is not possible for the principal to fully specify it in the contract; or because the task is simply too difficult to measure properly. Most contractual relationships contains such tasks, as for example a school teacher, apart from learning the pupils how to read and write, is also expected to provide them with a more general education; a nursing assistant should both deliver the basic physical care for a patient as well as caring for their mental health; or that the manager of a firm is expected focus on the long run profit and reputation of the firm as well as the short term earnings. The multitasking problem and its relation to incentive schemes has been examined in a number of earlier papers. Eggleston (2005) and Kaarboe and Siciliani (2011) each present a model with multitasking in the sense that the agent can perform two activities. Both activities contributes to the output received by the principal, but a binding contract can only be written on one of them. The effect of offering the agent a piece rate for undertaking the activity which can be contacted upon is ambiguous as it will lead the agent to neglect the other 
activity. Whether or not the stronger incentives ${ }^{1}$ to the agent is beneficial or not, will depend on the importance of the verifiable and non-verifiable dimensions. Both Eggleston and Kaarboe and Siciliani define the agent's performance as the level of effort exerted, and hence the performance measurement is a noisy measure of this level. Our analysis provides an extension to this as, in addition to the possibility to reward the agent with a piece rate for one of two possible activities, the principal can also reward the agent based on an imperfect measurement of the outcome achieved as a result of the activities. This allows us both to examine the effect of the principal only being able to reward some types of effort directly through the contract and the effect of an overall performance measurement of the agent's activities.

The second observation that drives this paper is that in many instances of contracting the agent, whether he be a CEO or a public servant, may also learn more about his capability of handling the inputs during the actual production process. To quote Holmström (1979, p. 88): "One unrealistic feature [of our economic model] is the assumption that the agent chooses his action having the same information as the principal [...] Commonly this will not be the case. After the sharing rule is fixed, the agent will often learn something new about the difficulty of his task or the environment in which it is to be performed." Indeed, in many cases we would expect the agent to acquire new information about the tasks in the production only while undertaking them. The teacher only learns the level of comprehension in a class while teaching them, a doctor only learns the difficulty of an operation while performing it, and the contractor only learns the difficulty of building a new bridge while actually building it. As an employee is typically much more familiar with the details of a task than is his superior, and because the knowledge acquired by the agent may only be obtained by experience in the task, it can be impossible for the principal to acquire the knowledge herself and for the agent to pass it on to the principal. The knowledge can therefore be specific in the sense of Jensen and Meckling (1992). A string of the literature has focussed on how to reward the agent in order to make him use the knowledge he might possess. Baker (1992) shows that if the agent is asymmetrically informed about how his actions affects the value to the principal, it may indeed be more optimal for a principal to let the agent choose actions freely and then pay him according to a performance measure. Prendergast (2002) extends this analysis by considering a situation where the principal can choose between assigning a task to the agent and monitor the agent's use of inputs, or to let the agent choose the task himself and then monitor the agent's output through a performance measurement. Prendergast concludes that the principal should rely more and more on the performance measurement when the agent's ability to choose

\footnotetext{
${ }^{1}$ The strength of the incentives refers to the level of wage to the agent that is depending on a specific parameter, in this case the level of effort the agent puts into an activity.
} 
a desirable project becomes more important to the principal. Our analysis differs from those of Baker and Prendergast in that we do not consider the agent's ability to choose between different projects, but rather to allocate an efficient amount of effort into an activity because he is asymmetrically better informed about his own productivity than is the principal. Further, we also allow the principal to reward both via a piece rate for effort in a specified activity and via a performance measurement. To reward the agent in this way is a often use method in practice, for example in Great Britain (Gillam et al., 2012) where general practitioners are rewarded both based on treatment delivered to the patients (efforts) and based on the health care results obtained (performance). Raith (2008) makes a similar analysis with rewards for both activities and a performance measure and notes that even though the agent receives his any private information to be used for free, he will have no incentives to use this knowledge without direct incentives to do so in the form of a reward for a 'good' performance. We further extends Raith's analysis by considering a situation where the agent can perform two types of effort of which one can be measured while one cannot be measured by the principal.

The third observation driving our analysis is that actual performance in many instances is impossible to measure. The use of performance based contracts is therefore, unfortunately, not without problems. Indeed, even though many of the findings in the literature show mostly positive results from implementing performance based contracting (Greene and Nash, 2009; Gravelle et al., 2010), there are negative effects as well as the public sector can generally suffer from too expensive or insufficiently clear indicators for performance (Weibel et al., 2010; Siva, 2010; Langbein, 2010). In many instances it will not be possible to find an objective measurement, and a principal can only observe a noisy indicator of the agent's actual performance. Domberger and Jensen (1997) point out that even within relatively simple tasks it can be problematic to determine whether or not "good performance" has been achieved. Typically it will be a subjective observation. Hence, the actual performance that the principal wishes the agent to achieve can be too difficult to fully describe in a contract. As noted by Baker (1992), it is not difficult to find some kind of indicator for performance that is related to the actual objective of the principal. National testing of pupils in schools seeks to establish whether or not they have received a good education or not, but these test may not capture the more elaborate part of education, e.g. how to behave properly or how to function socially. Even so, Baker (2000) shows that the use of performance measurements may induce stronger incentives to the agent to perform better, even if these measurements are imperfect. We capture this feature by letting the performance measurement used in the contract between the principal and the agent be only imperfectly correlated with the principal's objective, even to the point where there is approximately no correlation at all. 
Our basic findings are that when a principal can choose between rewarding the agent directly for a specific effort or rewarding the agent via an imperfect measurement of the output delivered, it will be optimal to at least partially reward for the performance. This result arises for two reasons; first because the agent can possess specific knowledge and a reward for performance induces him to make use of it in the interest of the principal; secondly because there can be multitasking issues in the contract, which are diminished by the use of performance measurement. A performance contract will be increasingly beneficial to the principal when either of these issues becomes more important in the contractual relationship.

The remainder of this paper is organised as follows: Section 2 will introduce the framework to our model, while section 3 will present a baseline contract between the principal and the agent. We extend this contract in section 4 by introducing a performance measurement. Further, in section 5 we will discuss some implications of the model and their relation to the existing literature. Interestingly, our basic finding are also closely related to a strictly different string of literature, that of Incomplete Contracts, which will be discussed in section 6 . Finally, section 7 will provide some concluding remarks.

\section{Model}

We consider a setting involving a principal $(P)$ and a single agent $(A)$. The agent possesses private knowledge about his own productivity. We assume that this knowledge is specific in the sense of Jensen and Meckling (1992), i.e. that the agent is not able to communicate this knowledge to the principal as the cost of doing so is infinitely high. However, to avoid an adverse selection problem, we assume that the agent receives his private knowledge after the contract has been signed, or in other words; the agent only learns his own productivity when he is about to perform the task. As an example, consider a doctor (the agent) who is hired by a hospital manager (the principal) to treat a patient. The optimal treatment can only be determined when the doctor examines the patient. Hence the principal will have to employ the doctor before the correct treatment can be known. Alternatively, the schoolteacher may not know how easy or difficult it will be to teach a given class until the teaching has begun. For a third example, consider the developer, who is hired to construct a bridge. When the contract with the principal is signed, the developer may have some expectation about his productivity in the task, but it is only when the construction of the bridge is about to begin and further examinations of the building sight has been carried out that he learns his actual productivity. Further, we assume there are situations which the principal cannot specify in the contract. The principal is thus able to perfectly specify some activities for the agent to perform 
(e.g. working hours or the number of patients treated at a medical clinic), but unable to specify other activities (e.g. general care for patients). The contract is thereby assumed to be incomplete in the sense that the agent can perform some actions, which the principal cannot contract upon. This assumption is well in line with both the literature of Multitasking (Holmström and Milgrom, 1991) and the general assumptions within Incomplete Contracting (see e.g. Hart, 1995 or Hart et al., 1997), both of which assumes that most contracts are incomplete with regard to the agent's possible efforts. As pointed out by especially the literature of health economics, the agent can have altruistic motives such he cares about the output to the principal, i.e. cares about delivering a good quality of service (Eggleston, 2005). These altruistic motives can be considered in several ways. Within the health care sector it is often assumed that the personal cares about the health of a patient - in many countries a doctor will e.g. take the Hippocratic Oath. An alternative understanding of the motives can be an agent caring about his future reputation after the contract with the principal has ended. Delivering a good output to the principal is likely to improve this reputation, and as such the altruistic term in the model below is general enough to also include a 'future effect' for the agent.

The contract offered by the principal specifies the payments to the agent for exerting effort or meeting targets for performance. We ignore the possibility of negotiations between the parties, so the agent can only accept or reject the principal's proposed contract. Both the agent and the principal are assumed to be risk neutral. The agent is, however, protected by limited liability and hence he must in all cases receive a non-negative wage. In spite of the assumption of risk-neutrality, this gives the model somewhat the same features as a risk-averse agent, as the principal cannot punish the agent for poor performance

We draw upon the setting of Raith (2008), but expands his model by adding a second, non-contractable input, and by removing a number of assumptions of symmetry in the model.

In order to produce an output to the principal the agent can provide effort at a personal cost into two kinds of activities. ${ }^{2}$ Output to the principal, $Y$, is stochastic and can take the values 0 or 1, i.e. a 'bad' and a 'good' outcome, respectively. ${ }^{3}$ The probability that $Y=1$ is given by

$$
\operatorname{Pr}(Y=1)=\min \left\{e_{1} \theta+e_{2} \varphi, 1\right\}
$$

where $e_{i}$ is the level of effort exerted in activity $i$, and $\theta$ and $\varphi$ are the agent's productivities for activity 1 and 2, respectively. The productivities

\footnotetext{
${ }^{2}$ Effort is to be understood in the most general way and can be taken to mean a wide range of actions available to the agent. Hence the efforts can both mean separate work processes or different actions within the same process.

${ }^{3} \mathrm{~A}$ number of the parameters in our analysis are modelled as discrete cases, which significantly simplifies calculations.
} 
are, however, uncertain in the sense that $\theta$ and $\varphi$ either are high $\left(\theta_{H}, \varphi_{H}\right)$ or low $\left(\theta_{L}, \varphi_{L}\right)$ with $\theta_{H}, \theta_{L}=\left(1 \pm v_{1}\right) \bar{\theta}$ and $\varphi_{H}, \varphi_{L}=\left(1 \pm v_{2}\right) \bar{\varphi}$, where $v_{i}>0$ measures the degree of variation (i.e. uncertainty) in the productivity and $\bar{\theta}, \bar{\varphi}$ are the mean productivities. These mean productivities are what both parties expect when signing the contract, after which the agent learns whether his actual productivities are actually higher or lower than this. Each outcome is equally likely such that $\operatorname{Pr}\left(\theta=\theta_{H}\right)=\operatorname{Pr}\left(\theta=\theta_{L}\right)=\operatorname{Pr}(\varphi=$ $\left.\varphi_{H}\right)=\operatorname{Pr}\left(\varphi=\varphi_{L}\right)=\frac{1}{2}$. After the contract is signed, the agent receives a set of private signals, $s^{i}=s_{H}^{i}, s_{L}^{i}$, about the actual size of $\theta$ and $\varphi$. The probability that the agent receives the correct signal is given by $\operatorname{Pr}\left(s_{H}^{1} \mid\right.$ $\left.\theta_{H}\right)=\operatorname{Pr}\left(s_{H}^{2} \mid \varphi_{H}\right) \operatorname{Pr}\left(s_{L}^{1} \mid \theta_{L}\right)=\operatorname{Pr}\left(s_{L}^{2} \mid \varphi_{L}\right)=\frac{1+k}{2}$. The agent's level of knowledge about his own productivity is captured in $k \in[0 ; 1]$, such that when $k=1$ he is perfectly informed and when $k=0$ he is completely uninformed and receives a signal by random.

The agent's utility is given by

$$
U_{A}=\alpha Y+W-\frac{c_{1}}{2} e_{1}^{2}-\frac{c_{2}}{2} e_{2}^{2}-d e_{1} e_{2}
$$

Here the term $\alpha Y$ captures any altruistic behaviour of the agent. $W$ is wages to be paid to the agent (described further below). $\frac{c_{i}}{2} e_{i}^{2}$, with $c_{i}>0$ for $i=$ 1,2 , is the agent's disutility from exerting effort $e_{i}$, and $d$ measures a spillover cost of engaging in both activities at the same time. As the two types of effort can be substitutes $(d>0)$, complements $(d<0)$ or independent $(d=0)$, he may incur an additional cost or benefit when providing both. We make the restrictions that $c_{i}>0$ and $c_{i}>|d|$ for $i=1,2$. The latter restriction ensures that the cost of effort should be the most significant part of the agent's cost function. When the efforts are complements it is thus possible to reduce total costs, although not to the point where the costs vanish.

The principal's utility is given by

$$
U_{P}=V Y-W
$$

where $V$ is the principal's value of receiving a good output.The higher the value, the more important is a good output compared to a bad. We assume that $V>\alpha$, such that the principal's valuation of a good output is higher than the value of the agent's altruistic motives.

The principal can choose to base the contract on the 'inputs' provided by the agent in the form of the level of effort that the agent allocates into an activity. We then extend the analysis by letting the principal base the 
contract on the 'output' resulting from the agent's provided effort. ${ }^{4}$ When the principal specifies 'inputs', we assume that she can fully observe and reward the agent's choice of effort in activity 1, while effort in activity 2 cannot be observed. Hence a binding contract can potentially be written on $e_{1}$, while no contract can be written based on $e_{2}$. It is worth noticing that a binding contract on a level of effort will never induce the agent to take any information he might possess about his own productivity into account.

We restrict our attention to a contract with a wage scheme that is linear parameters. This wage scheme for $e_{1}$ corresponds to e.g. an hourly wage for working hours dedicated to an activity and is motivated by its common use in practise.

The agent's wage is thus given by

$$
W=F+e_{1} \beta_{e}
$$

where $F$ is a fixed payment. As both $e_{1}$ can assume the value 0 (though it cannot be negative), the assumption of limited liability for the agent requires $F \geq 0$ as we must always have $W \geq 0$. We normalise the agent's outside option to $\underline{U_{A}}=0$ and can therefore set $F=0$.

The timing of the game is as follows

(i) The principal offers a contract specifying $W$ to the agent

(ii) The agent accepts or rejects the contract

(iii) The agent's productivities is realised and the agent receives a set of private signals

(iv) The agent chooses $e_{1}$ and $e_{2}$

(v) The output to the principal is realised

(vi) The agent receives payments according to the contract

All of this is common knowledge and the only source of uncertainty is that the agent is privately informed about $\theta$ and $\varphi$.

\section{The baseline contracts}

We first consider a baseline contract where the agent is only paid a fixed payment, and where the principal thus rely solely on the altruistic behaviour

\footnotetext{
${ }^{4}$ The terms 'input' and 'output' are to be understood in a broad sense, where they cover a range of different activities performed by the agent and more or less abstract results from these activities. An exact definition of what is input and what is output will therefore depend on the context; first of all because in many settings the output from one production will be the input in another production; and secondly, because it will depend on the perspective. For example, in the case of a physician operating on a patient, the physician's time spend can be understood as an input to the operation, which is in itself an output. An alternative perspective could be that the physician's time is only an input to the actual output; the patient's health, and that the result of the operation is only an imperfect indicator of this.
} 
of the agent to induce him to exert effort. Throughout the analysis we focus on an interior solution such that $0 \leq e_{1} \theta+e_{2} \varphi \leq 1$ and hence $E[Y]=$ $e_{1} \theta+e_{2} \varphi$.

In this case the agent's expected utility will be given by

$$
E\left[U_{A}^{B}\right]=\alpha\left(e_{1} \widehat{\theta}+e_{2} \widehat{\varphi}\right)-\frac{c_{1}}{2} e_{1}^{2}-\frac{c_{2}}{2} e_{2}^{2}-d e_{1} e_{2}
$$

where $\widehat{\theta}$ and $\widehat{\varphi}$ are the agent's own expectations about his productivity given the signals he received (for a precise definition of these, see the appendix). Maximizing the agent's utility with respect to the effort levels leads to Proposition 1

Proposition 1. Offering a contract with $W=F$ the agent will optimally choose

$$
e_{1}^{B}=\frac{\alpha}{c_{1} c_{2}-d^{2}}\left(c_{2} \widehat{\theta}-d \widehat{\varphi}\right) \text { and } e_{2}^{B}=\frac{\alpha}{c_{1} c_{2}-d^{2}}\left(c_{1} \widehat{\varphi}-d \widehat{\theta}\right)
$$

The agent's altruistic behaviour will make him exert some effort into the production, and the costs and productivity will make him allocate effort into the least costly and/or most productive task. It is also noteworthy that the agent to some extend uses his private knowledge about the productivities because of his altruism. The first best solution (defined as the sum of the principal's and the agent's utilities) would, however, be

$$
e_{1}^{F B}=\frac{\alpha+V}{c_{1} c_{2}-d^{2}}\left(c_{2} \widehat{\theta}-d \widehat{\varphi}\right) \text { and } e_{2}^{F B}=\frac{\alpha+V}{c_{1} c_{2}-d^{2}}\left(c_{1} \widehat{\varphi}-d \widehat{\theta}\right)
$$

It is therefore clear that when relying solely on altruism the agent will exert too little effort as he does not include the principal's valuation of the output. One widely used way of inducing the agent to exert more effort is to reward him directly for effort. The classical problem in this situation is, however, that the principal is unlikely to be able to directly reward all types of effort that the agent can exert. We therefore next consider the wage scheme $W=$ $F+\beta_{e} e_{1}$, where the principal can reward the agent for effort in activity 1 but not in activity 2 . This changes the agent's utility function slightly such that the expected utility now is given by

$$
E\left[U_{A}^{E}\right]=\alpha\left(e_{1} \widehat{\theta}+e_{2} \widehat{\varphi}\right)+e_{1} \beta_{e}-\frac{c_{1}}{2} e_{1}^{2}-\frac{c_{2}}{2} e_{2}^{2}-d e_{1} e_{2}
$$

Maximizing this will result in efforts

$$
\begin{aligned}
& e_{1}^{E}=\frac{1}{c_{1} c_{2}-d^{2}}\left[\alpha\left(c_{2} \widehat{\theta}-d \widehat{\varphi}\right)+c_{2} \beta_{e}\right] \text { and } \\
& e_{2}^{E}=\frac{1}{c_{1} c_{2}-d^{2}}\left[\alpha\left(c_{1} \widehat{\varphi}-d \widehat{\theta}\right)-d \beta_{e}\right]
\end{aligned}
$$

As a result of the agent's efforts, the principal will set $\beta_{e}$ to maximize $E\left[U_{P}^{E}\right]=E\left[e_{1}^{E} \theta+e_{2}^{E} \varphi\right] V-\beta_{e} e_{1}^{E}$, which will lead to Proposition 2 
Proposition 2. Offering a contract with $W=F+\beta_{e} e_{1}$ to the agent, the principal can most optimally choose

$$
\beta_{e}^{E}=\frac{V-\alpha}{2 c_{2}}\left(c_{2} \bar{\theta}-d \bar{\varphi}\right)
$$

This result show that the principal first of all will reward the agent more when the outcome is more important to the principal. Further, the relative sizes of the productivities will affect the wages such that it increases in productivity for activity 1 and decreases in productivity for activity 2 . The principal thus decides whether or not she wishes to motivate the agent to exert effort into activity 1 by seeing which activity has the greatest chance of creating a good output to the principal. Interestingly, the cost of effort in activity 1 does not affect the wage. Rather, it will increase in cost of effort in the other activity $\left(c_{2}\right)$ and decrease in the spillover cost $(d)$. The intuition for the first is that whenever $c_{2}$ is relatively high it will motivate the agent to focus more on activity 1 , and hence a reward for this activity will motivate the agent even further. If, on the other hand, $c_{2}$ is relatively low it can be beneficial for the principal to allow the agent to focus more on activity 2 and hence the wage for effort in activity 1 is lowered. A higher spillover cost will make the principal refrain from rewarding the agent more since this will also make him dedicate less effort into activity 2. Further, the result shows that the agent private knowledge has no effect on the wage offered by the principal for providing effort as this wage is paid per unit of effort whichever effect this effort has on the output to the principal, and whatever knowledge the agent might hold about this. In other words, even though the principal might motivate the agent to exert effort through this wage, it does not induce the agent to use private knowledge in the interest of the principal. Finally, it is interesting to note that for activities that work as complements, the principal will increase the wage since a higher level of effort in activity 1 will also motivate the agent to increase the level of effort in activity 2 . To see this, we find the expected level of effort exerted by the agent, given the wage $\beta_{e}^{E}$.

Proposition 3. Given the wage $\beta_{e}^{E}$, the agent will in expectation choose

$$
\begin{aligned}
& E\left[e_{1}^{E}\right]=\frac{\frac{1}{2}(\alpha+V)}{c_{1} c_{2}-d^{2}}\left(c_{2} \bar{\theta}-d \bar{\varphi}\right) \text { and } \\
& E\left[e_{2}^{E}\right]=\frac{\alpha}{c_{1} c_{2}-d^{2}}\left(c_{1} \bar{\varphi}-d \bar{\theta}\right)-\frac{d}{2 c_{2}} \cdot \frac{V-\alpha}{c_{1} c_{2}-d^{2}}\left(c_{2} \bar{\theta}-d \bar{\varphi}\right)
\end{aligned}
$$

The use of a direct reward for effort in activity 1 increases the level of effort in this activity and brings it closer to the first best level. To see this, note that as $V>\alpha$ by assumption, we also have $\frac{1}{2}(V+\alpha)>\alpha$. The cost of this, however, is that the effort in activity 2 decreases when the two activities are substitutes $(d>0)$. This is the classical prediction within the 
multitasking literature; rewarding one effort will make the agent substitute away from the unrewarded effort and instead focus on the activity that is rewarded. If $d<0$ the two activities are complements, and in this case the level of effort in activity 2 will increase as well. So in this case it is possible to increase both efforts simply by rewarding one of them.

\section{Performance measurement}

We now introduce a performance measurement into the model above. The principal can therefore now also reward the agent for delivering an output. However, in line with Baker (1992) we assume that the principal is not able to use her own objective function as a measurement of the performance of the agent. As Domberger and Jensen (1997) points out, it can be difficult to define e.g. 'good quality' even in a relatively simple task such as cleaning. Often the only way to measure the quality will be to have someone inspecting the outcome, and then determine whether it can be classified as good quality or not. Even if the principal has a relatively clear idea of what is "good quality", she must specify it in concrete and measurable units in the contract. If it is not possible to specify everything to a degree where a third party (e.g a court) can verify whether the specifications in the contract has been met or not, then the contract is in effect incomplete. To avoid the incomplete contract, the principal will instead rely on an imperfect - but contractable - indicator of $Y$, here denoted by $y$.

The indicator $y$ takes the values 0 or 1 (similarly to $Y$ ). The probability that the indicator is trustworthy such that $y=Y$ is given by

$$
\operatorname{Pr}(y=Y)=1-\frac{\gamma}{2}
$$

The parameter $\gamma \in] 0,1[$ measures the error in the indicator $y$. If $\gamma=1$ the indicator is completely random and thus completely uninformative for the principal. On the other hand, if $\gamma=0 Y$ can be perfectly measured by $y$. In these two extreme cases a contract will be based solely on either $e_{1}$ or $y$, and many of the results of the model are trivial. Hence we restrict our attention to the intermediate interval $0<\gamma<1$.

We still focus on a linear wage scheme to the agent such that he will only receive payment for providing the output if the quality parameters specified in the contract are met, i.e. if $y=1$. In other words, the agent only receives this part of his wage when the indicator for performance is satisfied. If it is not met, the agent receives no reward for performance. As the indicator for performance is imperfect, $y=1$ does not necessarily imply that the principal actually received a 'good' output, i.e. $Y=1$. The principal still has the option to reward the agent for providing effort and by offering a fixed 
payment. The agent's new wage scheme is therefore given by

$$
W^{\prime}=F+e_{1} \beta_{e}+y \beta_{y}
$$

Like previously, the fixed wage offers no incentives to the agent while limited liability keeps it non-negative. So again we have $F=0$.

Assuming that we have an interior solution such that $e_{1} \theta+e_{2} \varphi \in[0 ; 1]$, the expected value of $y$, conditional on $e_{1}, e_{2}, \theta$ and $\varphi$, can be found as the probability of the good outcome being achieved times the probability of the indicator being correct, plus the probability of the bad outcome being achieved times the probability of the indicator showing the wrong outcome. The remaining probability combinations all result in $y=0$ and are therefore not included in the calculations below.

$$
E[y]=\operatorname{Pr}(Y=1) \operatorname{Pr}(y=Y)+\operatorname{Pr}(Y=0) \operatorname{Pr}(y \neq Y)
$$

Inserting the probabilities above yields

$$
E\left[y\left(e_{1}, e_{2}, \theta, \varphi\right)\right]=\frac{\gamma}{2}+(1-\gamma)\left(e_{1} \theta+e_{2} \varphi\right)
$$

In order to find the optimal contract for the principal to offer to the agent, we need to bring the above equations together. Inserting (3), the agent's wage, and (6), the expected value of $y$, into (1), the agent's utility function, we find the agent's expected utility:

$$
\begin{aligned}
E\left[U_{A}^{P}\right] & =\alpha\left(e_{1} \widehat{\theta}+e_{2} \widehat{\varphi}\right)+e_{1} \beta_{e} \\
& +\beta_{y}\left[\frac{\gamma}{2}+(1-\gamma)\left(e_{1} \widehat{\theta}+e_{2} \widehat{\varphi}\right)\right]-\frac{c_{1}}{2} e_{1}^{2}-\frac{c_{2}}{2} e_{2}^{2}-d e_{1} e_{2}
\end{aligned}
$$

The agent chooses $e_{1}$ and $e_{2}$ in order to maximise this, which yields

$$
e_{1}^{*}=\frac{1}{c_{1} c_{2}-d^{2}}\left[\left(\alpha+\beta_{y}(1-\gamma)\right)\left(c_{2} \widehat{\theta}-d \widehat{\varphi}\right)+c_{2} \beta_{e}\right]
$$

and

$$
e_{2}^{*}=\frac{1}{c_{1} c_{2}-d^{2}}\left[\left(\alpha+\beta_{y}(1-\gamma)\right)\left(c_{1} \widehat{\varphi}-d \widehat{\theta}\right)-d \beta_{e}\right]
$$

Each of the effort decrease in own costs and increase in the cost of the other effort. Likewise, each effort increase in own productivity and decreases in the productivity of the other effort. Like in the previous cases, costs and productivities thereby make the agent allocate effort most optimally between the two activities. The wage for effort makes the agent focus on the rewarded activity, while it also increases the level of effort in the other activity if the two activities are complements. Rewarding for performance will increase the effort that most efficiently makes a good output to the principal most likely. Further, it decreases in the measurement error of performance. Also, note 
that if $\gamma=1$, any payment for output will not affect the agent's choice of effort as $y$ will indicate a 'good' or 'bad' outcome by random.

Given the agent's choice of effort, the principal will set $\beta_{e}$ and $\beta_{y}$ to provide incentives to the agent.

Proposition 4. Offering a linear contract of the form $W^{\prime}=e \beta_{e}+y \beta_{y}$, the optimal contract which the principal can offer to the agent is

$$
\beta_{e}^{P}=\frac{\gamma\left(c_{1} c_{2}-d^{2}\right)\left(c_{2} \bar{\theta}-d \bar{\varphi}\right)}{4(1-\gamma) \eta}
$$

for providing effort, and

$$
\beta_{y}^{P}=\frac{V-\alpha}{2(1-\gamma)}-\frac{\gamma\left(c_{1} c_{2}-d^{2}\right) c_{2}}{4(1-\gamma)^{2} \eta}
$$

for providing the output.

With $\eta=\left(c_{2}^{2} k^{2} v_{1}^{2} \bar{\theta}^{2}+c_{1} c_{2} k^{2} v_{2}^{2} \bar{\varphi}^{2}+c_{1} c_{2} \bar{\varphi}^{2}-d^{2} \bar{\varphi}^{2}\right)$

Proof. See the appendix

Compared to the previous situation where the principal can only use rewards for effort in activity 1, a number of results are worth emphasising; First of all, $c_{1}$ now directly affect the wage for effort and will generally lead to an increase. As the agent now has the possibility to receive compensation without using effort in activity 1 (through the performance payment), the principal needs to compensate him when $c_{1}$ increases. As previously, the effect of, $d$, depends on whether the activities are substitutes or complements, such that for substitutes $\beta_{e}$ decreases in $d$ and for complements it increases in the numerical value of $d$. For the performance wage there will generally be an increase in $\beta_{y}$ when the numerical value of $d$ increases so it either becomes more important to make the agent focus on the most efficient activity (in the case of substitutes) or it simply becomes important to motivate the to use as much effort as possible (complements). Further, neither the principal's valuation of the output nor the agent's altruism affect the wage for effort any longer, while both affect the wage for performance. An increase in the principal's valuation will lead to an increase in performance wage as the achievement of a good output becomes more important, and hence the agent's motivation is increased. Conversely, if the agent's altruistic motives becomes stronger payment will be decreased. The inclusion of the performance measurement further means that the wage for effort is affected by a number of other factors, such that it increases in $\gamma$ while it decreases in $k$, $v_{1}$ and $v_{2}$. When the measurement error in the output increases, payment for effort becomes relatively more beneficial for the principal as the signal for 'good' or 'bad' output becomes less certain. A second effect is that measurement error allocates risk to the agent, which also makes it preferable 
for the principal to focus more on payment for effort which offers no risk to the agent. When the agent's knowledge becomes more important, the principal will provide stronger incentives to the agent in order to motivate him to use this knowledge and thereby decrease $\beta_{e}$ while increasing $\beta_{y}$. Notice that the agent's knowledge becomes more important both when $k$ and $v_{i}$ increases, as the former makes the agent better informed and the latter means a larger variation in productivity, which makes the a 'correct' allocation of effort more important, thereby making any knowledge the agent might possess more important. Further, like in the case with reward only for effort, $\beta_{e}$ increases in the agent's productivity in activity 1 and decreases in that of activity 2 . For the performance payment the effect is more universal as $\beta_{y}$ decreases in both productivities since higher productivity means that it becomes increasingly easy for the agent to achieve a 'good' output. This in turn decreases the agent's costs and thereby the risk he has to undertake when he is awarded for the output.

Having found the optimal wages that the principal can offer to the agent given the linear wage scheme, we can find the levels of effort that the agent will exert.

Proposition 5. Using a linear wage scheme $W^{\prime}=e_{1} \beta_{e}+y \beta_{y}$, with $\beta_{e}=\beta_{e}^{P}$ and $\beta_{y}=\beta_{y}^{P}$ as defined in (9) and (10), the agent will in expectations provide efforts

$$
E\left[e_{1}^{P}\right]=\frac{\frac{1}{2}(V+\alpha)}{\left(c_{1} c_{2}-d^{2}\right)}\left(c_{2} \bar{\theta}-d \bar{\varphi}\right)
$$

and

$$
E\left[e_{2}^{P}\right]=\frac{\frac{1}{2}(V+\alpha)}{\left(c_{1} c_{2}-d^{2}\right)}\left(c_{1} \bar{\varphi}-d \bar{\theta}\right)-\frac{\gamma\left(c_{1} c_{2}-d^{2}\right)}{4(1-\gamma) \eta}
$$

with $\eta=\left(c_{2}^{2} k^{2} v_{1}^{2} \bar{\theta}^{2}+c_{1} c_{2} k^{2} v_{2}^{2} \bar{\varphi}^{2}+c_{1} c_{2} \bar{\varphi}^{2}-d^{2} \bar{\varphi}^{2}\right)$

Proof. Inserting (9) and (10) into (7) and (8) and taking the expected value leads directly to Proposition 5

The use of a performance measurement does not in expectation lead to a change in the level of effort in activity 1 compared to the case where wage is solely based on effort in activity 1 (i.e. compared to Proposition 3). Rather, the principal can already achieve the sub-optimal level through the wage for effort, and hence she substitutes between the two wages to keep this level. The performance measurement will, however, change the level of effort in activity 2 . When the agent is only rewarded for effort in activity 1 , productivity in activity 2 only has an effect only as far as the agent has altruistic motives and when the agent's activities are complements. The performance contract adds to these effects by further motivating the agent to use a productive activity 2 through the pay for performance. The effect will, through 
the size of the performance payment, depend on the error in the measurement and on the importance of the agent's knowledge. There will therefore in expectations be a decrease in effort when the error $(\gamma)$ increases, and an increase when either the agent becomes more knowledgeable ( $k$ increase) or when the variation in productivity $\left(v_{i}\right)$ increases and his knowledge thereby becomes more important. Note that in the special case of $\gamma=0$, i.e. there is no error in the performance measurement, the second term in $E\left[e_{2}^{P}\right]$ disappears and the two effort levels will each be half the first best level. This result stems from (9) and (10), where it is clear that when performance can be measured perfectly, the principal will set $\beta_{e}=0$ and only reward the agent through $\beta_{y}$.

As the expected output to the principal is depending directly on the levels of effort exerted by the agent, it is generally in the principal's interest to induce the agent to provide as much as possible. As noted above, the principal cannot in expectations increase the level of effort in activity 1 , but the performance measurement will still motivate the agent to use his private knowledge about the actual size of the productivities in each task. Introducing a performance measurement to the contract scheme offered to the agent further means that the principal has an indirect way to reward the agent for effort in activity 2. Despite of this, there are a number of factors who determine whether or not the expected level of effort will increase compared to the situation with payment for effort in activity 1 .

Proposition 6. Including a performance wage in the contract will, in expectations, lead the agent to increase effort in activity 2 , such that $E\left[e_{2}^{E}\right]<$ $E\left[e_{2}^{P}\right]$, if

$$
\frac{\gamma}{(1-\gamma)} \frac{\left(c_{1} c_{2}-d^{2}\right) c_{2}}{2 \bar{\varphi} \eta}<V-\alpha
$$

with $\eta=\left(c_{2}^{2} k^{2} v_{1}^{2} \bar{\theta}^{2}+c_{1} c_{2} k^{2} v_{2}^{2} \bar{\varphi}^{2}+c_{1} c_{2} \bar{\varphi}^{2}-d^{2} \bar{\varphi}^{2}\right)$

A higher $V$ means that the principal values the output more, and as can be seen from Propositions 2 and 4 this result in higher wage for effort in activity 1 when this is the principal's only reward mechanism, while when she can use a performance measurement she will instead reward performance. Both of these effects increase the difference in $E\left[e_{2}\right]$ between the two cases. The effect of $\alpha$ is similar, although reversed. When the agent thus is very altruistic - to the point where he cares as much of the output as the principal does - the change in effort level is not likely to be large, as much effort is already provided without monetary rewards for activity 2 . The difference in effort levels will further depend on the usefulness of the performance measurement. If the measurement is imprecise ( $\gamma$ is high), the agent's knowledge limited ( $k$ is low) or unimportant ( $v_{i}$ is low), the effect of implementing the performance measurement can in expectations be a lower level of effort in activity 2 . On the other hand, a higher productivity induces the agent to exert 
effort as this makes payment for good performance more likely, and hence in expectations increase the level of effort under performance contracting. When the agent's altruism is not enough to motivate to exert effort into activity 2 , and when this effort is important to the principal, the use of performance measurements can help decrease the problem of multitasking and induce the agent to also focus on activity 2 .

\section{$5 \quad$ Implications}

Our results are closely related to several strings of literature, first and foremost that of multitasking. The prediction within the literature, dating back to Holmström and Milgrom (1991), is that when contracts are incomplete in the sense that there are activities, which the principal cannot contract upon, but which are of importance to her, she should provide weaker incentives to the agent and instead monitor him. In their setting, it will then be possible for principal to make him exert even the non-contractable effort, if the agent is an employee of the principal. Our analysis is in line with this argument so far that the principal should not provide stronger incentives for any particular activity when multitasking is present. Instead of monitoring the agent's every action, the principal has the possibility to motivate him through the performance measurement. Hence, our result is the opposite of Holmström and Milgrom; when multitasking is important, the principal should incentivise the agent more, but do it via a performance measurement instead of a direct payment for effort.

More recent contributions to the multitasking literature, made by Eggleston (2005) and Kaarboe and Siciliani (2011), both compare a situation where the agent is only motivated through altruistic motives and a situation where the principal can reward the agent for effort in one of two available activities. Their results are inconclusive as a direct reward for one effort leads to an increased use of this, but at the same time the agent will decrease the other effort. The general prediction is that as the multitasking issue becomes more important, i.e. the non-contractable efforts has a greater effect, the principal should rely less on incentives to the agent. We take this analysis step further by also allowing the principal to use an imperfect measurement of the outcome to reward the agent. Therefore we reach a conclusion opposite to those of the analyses above; when multitasking is more important the principal should focus on the performance measurement and through this provide more incentives to the agent.

Another interesting discussion in the literature is about the relationship between the uncertainty in the principal-agent relationship and the wage based on performance measurement. Baker (1992) shows that as the uncertainty in the relationship increases, the principal should focus less on a performance measurement. Prendergast (2002) instead focusses on a sit- 
uation where the agent has private information about the uncertainty and shows that when the uncertainty increases, the principal should offer stronger incentives to the agent to use this knowledge by increasing the wage for the performance measure. In an interesting study, Baker and Jorgensen (2003) compares two situations with uncertainty in the model; one where the agent has some private information about his productiveness, and a second where both parties are equally uninformed. They show that the agent's private information about uncertainty will lead the principal to offer him a higher wage based on a performance measurement, while general uncertainty in the measurement has the opposite effect. Our model is closely related to that of Baker and Jorgensen as we allow for two for types of uncertainty; uncertainty in the performance measurement $(\gamma)$ and uncertainty in the productivity $( \pm v)$. The agent receives a private signal about the latter, while the former is a general uncertainty. We show that as the performance measurement becomes less informative about the agent's actual performance $(\gamma$ increases) the principal should lower the wage based on this measurement and instead focus on rewarding effort. If either the agent becomes better informed ( $k$ increases) or the variation in his productivity becomes greater ( $v$ increases), the principal should create incentives for the agent to utilise his special knowledge be rewarding him more based on the performance.

The use of a performance measurement therefore has the potential to both lessen the problem of multitasking in a principal-agent relationship. If the measurement is precise enough the problem can be removed completely. Further, it also helps the principal motivate the agent to use any expert knowledge he might possess about the work process in the interest of the principal.

Our results are applicable in many instances. An example could be within teaching where a teachers contract often will specify hours of teaching and preparation, but with little or no direct specification of the quality of teaching as this is extremely complicated to specify in details. In order to motivate teachers beyond their potentially altruistic motives to deliver good teaching to students many institutions have a 'Teacher of the year' award. Despite this typically being a relatively small bonus that is only given to a single teacher each year, it can potentially be sufficient motivation to improve the overall teaching quality. Further, a number of schooling systems have implemented performance measurements in the teachers' contracts such that they in part are paid depending on the students' test results (Figlio and Kenny, 2007).

Another application of our results is the use of Public-Private Partnerships (PPP). Where contracts between the public sector and private firms traditionally have been concerned with relatively simple tasks (cleaning and garbage collection are typical examples), the use of PPPs aims at involving 
the private sector in much larger and more complex activities. ${ }^{5}$ A key feature of PPP is that, apart from specifying a number of tasks to be performed, the contract also typically opens for a number of bonuses to the private partner for 'good' performances. Hence when writing on a more complicated task, where the issue of multitasking is more prone to exist, the contract seeks to take this into account by - among other initiatives - motivating the agent via bonus payments.

\section{Relation to Incomplete Contracts}

In the analysis above we have focussed on the agent being able to contribute to the production process with an activity that is desirable for the principal, but which the principal cannot specify in a contract. It is, however, not given that such non-contractable should be beneficial for the principal. Rather, it can be possible for the agent to undertake an activity that is beneficial for himself, but either of no importance or is directly harmful for the principal. Considering this possibility, our basic findings are directly related to a strictly different string of literature, namely that of Incomplete Contracting (henceforth IC).

In the basic setting within IC the principal and the agent sign a contract on a type of effort. However, the agent has the possibility to perform one or more additional types of effort that will affect both his own cost and the output to the principal. These effects are opposite, such that e.g. an increase in the quality of output to the principal increases the agent's cost, and a decrease in the agent's cost will decrease the value of the output to the principal. The first of these cases corresponds to the situation described in the model above, while the second is a situation where the agent has the possibility to 'cut corners' in the production, i.e. decrease the quality of the output to the principal and through this save costs (see for example Hart et al., 1997). Within the IC literature the agent is able to decrease the quality of output without breaching the contract because the output to the principal is too complex to be described explicitly in the contract. The underlying assumptions of IC are therefore quite similar to those we have described for our model, but we make the additional assumption that the principal is able to specify a performance measurement which can be contracted upon, and which is indirectly affected by the agent's activities through its (possibly poor) correlation with the actual output to the principal. However, where IC focusses on a renegotiation of the contract as the solution to the incompleteness of the contract, our focus is on the principal's design of an initial

\footnotetext{
${ }^{5}$ The precise distinction between traditional public outsourcing (or contracting out) and PPP is debatable. A key difference is, however, that in the latter case the private partner can both be asked to finance, construct, maintain, and possibly manage an otherwise public activity - for example a school (see for example Yescombe (2011)
} 
contract that seeks to take the agent's possibility of alternative activities into account.

Our model is directly applicable to the IC literature if we consider the case of $\varphi<0$ and $d<0$. The agent thus have the possibility to decrease own costs at the expense of a smaller probability of a good output being delivered to the principal. In the baseline contract of Proposition 3 the agent will only refrain from using this cost saving activity when his altruistic motives are strong enough. The principal therefore suffers the same problem as within IC since she cannot control the agent's alternative actions. It is however possible for the principal to motivate the agent through a performance contract. As is seen Proposition 4 both $d$ and $\varphi$ affects the performance wage only as quadratic terms and consequently it is their numerical size that is of importance. When either of $\varphi$ or $d$ increase numerically, it means that the non-contractable part of the work process becomes more important to one or both of the players. This will lead the principal to increase the wage based on the performance measurement in order to motivate the agent to focus more on delivering a good output to the principal and focus less on saving costs. The principal can therefore to a large degree compensate for the agent's possibility to act in his own interest through the use of a performance measurement without the need for renegotiations with the agent.

\section{Concluding remarks}

This paper studies the issue of multitasking in a context where the agent who holds private information about his own productivity in each task. We first show that the principal cannot motivate the agent to use any noncontractable effort nor any private knowledge through a traditional contract with wage depending on effort. The agent will only do this as far as he hold altruistic motives or when the two activities are complements. In other words; the agent will only take these issues into account when it is in his own interest to do so. We then investigate a contractual relationship where the principal can reward the agent both through a contract based on effort in some activities and through an imperfect measurement of the outcome to the principal. By basing the payment to the agent on a measurement that is at least partially correlated with the actual output to the principal, the agent will be motivated to act in the principal's interest during the production. Because of this, the performance contract can potentially remove the problem of multitasking because the agent becomes free to use whichever activity he chooses to achieve a good output to the principal, and thereby can also choose to use activities that the principal otherwise cannot specify in the contract. Further, as the agent's private knowledge is important for the output of the production, the performance measurement also helps motivate to use this when choosing how much effort to use and where to allocate it. 


\section{Appendix}

Proof of Proposition 4. As before, the principal's utility is given by $U_{P}^{P}=$ $V Y-W^{\prime}$. By inserting the agent's wage, (3), and the expected value of $y$, (6), into the utility function, we find the principal's expected utility:

$$
E\left[U_{P}\right]=\left[V-\beta_{y}(1-\gamma)\right] E\left[e_{1}^{*} \theta+e_{2}^{*} \varphi\right]-\beta_{e} e_{1}^{*}-\beta_{y} \frac{\gamma}{2}
$$

with $e_{1}^{*}$ and $e_{2}^{*}$ defined as in (7) and (8).

Using $\operatorname{Pr}(Y=1)=\min \left\{e_{1} \theta+e_{2} \varphi, 1\right\}$, assuming an interior solution and inserting (7) and (8), the expected value of $Y$ is

$$
\begin{aligned}
E[Y]= & E\left[e_{1}^{*} \theta+e_{2}^{*} \varphi\right] \\
= & \frac{\theta}{c_{1} c_{2}-d^{2}}\left[\beta_{y}(1-\gamma)\left(c_{2} \widehat{\theta}-d \widehat{\varphi}\right)+c_{2} \beta_{e}\right] \\
& +\frac{\varphi}{c_{1} c_{2}-d^{2}}\left[\beta_{y}(1-\gamma)\left(c_{1} \widehat{\varphi}-d \widehat{\theta}\right)-d \beta_{e}\right] \\
= & \frac{1}{c_{1} c_{2}-d^{2}}\left[\beta_{e}\left(E[\theta] c_{2}-E[\varphi] d\right)\right. \\
& \left.+\beta_{y}(1-\gamma)\left(E[\theta \widehat{\theta}] c_{2}-E[\theta \widehat{\varphi}] d+E[\varphi \widehat{\varphi}] c_{1}-E[\varphi \widehat{\theta}] d\right)\right]
\end{aligned}
$$

Next, we need to find the agent's belief $\widehat{\theta}$ and $\widehat{\theta}$ as a function of $s^{i}$. It is here convenient to express the productivities as $\theta=\left(1+\tau_{1} v_{1}\right) \bar{\theta}$ and $\varphi=\left(1+\tau_{2} v_{2}\right) \bar{\varphi}$, with $\tau_{i}= \pm 1$ and the signal as $s_{H}^{i}, s_{L}^{i}= \pm 1$. The agent's expectations of $\tau_{i}$ depending on the signals $s^{i}$ will then be $E\left[\tau_{i} \mid s^{i}\right]=$ $s^{i \frac{1+k}{2}}-s^{i \frac{1-k}{2}}=s k$ for each $i=1,2$. The agent's beliefs $\widehat{\theta}$ and $\widehat{\varphi}$ are then given by $\widehat{\theta}=\left(1+v_{1} s k\right) \bar{\theta}$ and $\widehat{\varphi}=\left(1+v_{2} s k\right) \bar{\varphi}$. As the principal does not receive any signal, her expectations are slightly different. The expectations of $\tau_{i}$ unconditional on $s^{i}$ are $E\left[\tau_{i}\right]=0$ and since any errors in the agent's signals are symmetric, we will also have $E\left[s^{i}\right]=0$. Using this, we find that $E[\theta]=E\left[\left(1+\tau_{1} v_{1}\right) \bar{\theta}\right]=\bar{\theta}, E[\varphi]=E\left[\left(1+\tau_{2} v_{2}\right) \bar{\varphi}\right]=\bar{\varphi}, E[\widehat{\theta}]=$ $E\left[\left(1+v_{1} s^{1} k\right) \bar{\theta}\right]=\bar{\theta}$ and $E[\widehat{\varphi}]=E\left[\left(1+v_{2} s^{2} k\right) \bar{\varphi}\right]=\bar{\varphi}$. Further, $E[\theta \widehat{\theta}]=$ $E\left[\left(1+v_{1} s^{1} k\right)\left(1+\tau_{1} v_{1}\right) \bar{\theta}^{2}\right]$ reduces to $E[\theta \widehat{\theta}]=\left(1+v_{1}^{2} k E\left[s^{1} \tau_{1}\right]\right) \bar{\theta}^{2}$ and for $E[\varphi \widehat{\varphi}]$ we have $E[\varphi \widehat{\varphi}]=E\left[\left(1+\tau_{2} v_{2}\right)\left(1+v_{2} s^{2} k\right) \bar{\varphi}^{2}\right]=\left(1+v_{2}^{2} k E\left[s^{2} \tau_{2}\right]\right) \bar{\varphi}^{2}$. For $s^{i}=\tau_{i}= \pm 1$ we have $s \tau=1$ and for $s \neq \tau$ we have $s \tau=-1$. The probability of the former event is $\frac{1+k}{2}$ and $\frac{1-k}{2}$ for the latter. We therefore have $E[s \tau]=1 \frac{1+k}{2}-1 \frac{1-k}{2}=k$ for both $i=1,2$ and thus $E[\theta \widehat{\theta}]=\left(1+v_{1}^{2} k^{2}\right) \bar{\theta}^{2}$ and $E[\varphi \widehat{\varphi}]=\left(1+v_{2}^{2} k^{2}\right) \bar{\varphi}^{2}$. Finally, we have $E[\varphi \widehat{\theta}]=E[\theta \widehat{\varphi}]=\bar{\varphi} \bar{\theta}$.

Inserting all the elements described above into (A.2), the expression re- 
duces to

$$
\begin{aligned}
E[Y]= & E\left[e_{1}^{*} \theta+e_{2}^{*} \varphi\right] \\
= & \frac{1}{c_{1} c_{2}-d^{2}}\left[\beta_{e}\left(\bar{\theta} c_{2}-\bar{\varphi} d\right)\right. \\
& \left.\quad+\beta_{y}(1-\gamma)\left(\left(1+v_{1}^{2} k^{2}\right) \bar{\theta}^{2} c_{2}-2 d \bar{\theta} \bar{\varphi}+\left(1+v_{2}^{2} k^{2}\right) \bar{\varphi}^{2} c_{1}\right)\right]
\end{aligned}
$$

Inserting (A.3) and the agents choice of effort, (7) and (8), into (A.1) and maximising it with relation to $\beta_{e}$ and $\beta_{y}$ will yield (9) and (10), respectively and thereby lead to Proposition 4 .

\section{References}

Baker, G. (1992). Contracts and Performance Measurement. Journal of Political Economy $100(3)$, pp. 598-614.

Baker, G. (2000). The Use of Performance Measures in Incentive Contracting. The American Economic Review 90(2), pp. 415-420.

Baker, G. and B. Jorgensen (2003). Volatility, noise, and incentives. Working paper, Harvard University.

Burgess, S. and M. Ratto (2003). The Role of Incentives in the Public Sector: Issues and Evidence. Oxford Review of Economic Policy 19(2), pp. 285-300.

Domberger, S. and P. Jensen (1997). Contracting Out by the Public Sector: Theory, Evidence, Prospects. Oxford Review of Economic Policy 13(4), pp. $67-78$.

Eggleston, K. (2005). Multitasking and mixed systems for provider payment. Journal of Health Economics 24 (1), 211-223.

Figlio, D. N. and L. W. Kenny (2007). Individual teacher incentives and student performance. Journal of Public Economics 91(5), pp. 901-914.

Gillam, S. J., A. N. Siriwardena, and N. Steel (2012). Pay-for-performance in the united kingdom: impact of the quality and outcomes framework - a systematic review. The Annals of Family Medicine 10(5), pp. 461-468.

Gravelle, H., M. Sutton, and A. Ma (2010). Doctor Behaviour under a Pay for Performance Contract: Treating, Cheating and Case Finding? The Economic Journal 120(542), F129-F156.

Greene, S. E. and D. B. Nash (2009). Pay for Performance: An Overview of the Literature. American Journal of Medical Quality 24(2), pp. 140-163. 
Hart, O. (1995). Firms Contracts and Financial Structure. Oxford University Press.

Hart, O., A. Shleifer, and R. W. Vishny (1997). The Proper Scope of Government: Theory and Application to Prisons. The Quarterly Journal of Economics 112(4), pp. 1127-1161.

Holmström, B. (1979). Moral Hazard and Observability. The Bell Journal of Economics 10(1), pp. 74-91.

Holmström, B. and P. Milgrom (1991). Multitask Principal-Agent Analyses: Incentive Contracts, Asset Ownership, and Job Design. Journal of Law, Economics, 83 Organization 7(1), pp. 24-52.

Jensen, M. C. and W. H. Meckling (1992). Specific and General Knowledge and Organizational Structure. In L. Werin and H. Wijkander (Eds.), Contract Economics, pp. 251-274. Oxford: Blackwell.

Kaarboe, O. and L. Siciliani (2011). Multi-Tasking, Quality and Pay for Performance. Health Economics 20(2), pp. 225-238.

Langbein, L. (2010). Economics, public service motivation, and pay for performance: Complements or substitutes? International Public Management Journal 13(1), 9-23.

Prendergast, C. (2002). The Tenuous Tradeoff Between Risk and Incentives. The Journal of Political Economy 110(5), pp. 1071-1102.

Raith, M. (2008). Specific knowledge and performance measurement. The RAND Journal of Economics 39(4), pp. 1059-1079.

Siva, I. (2010). Using the lessons of behavioral economics to design more effective pay-for-performance programs. The American journal of managed care 16(7), 497.

Weibel, A., K. Rost, and M. Osterloh (2010). Pay for performance in the public sector-benefits and (hidden) costs. Journal of Public Administration Research and Theory 20(2), 387-412.

Yescombe, E. R. (2011). Public-Private Partnerships: Principles of Policy and Finance. Butterworth-Heinemann. 